\section{New insight into non-healing corneal ulcers: iatrogenic crystals s}

\begin{abstract}
Aims To characterise and correlate crystalline precipitations implicated in non-healing corneal ulceration in two patients with a previous history of acanthamoeba keratitis. Materials and methods Persistence of acanthamoeba and secondary bacterial infection was excluded with negative corneal scrapes. Confocal microscopy identified crystal-like deposits within the corneal stroma. To investigate possible precipitating combinations, all concurrent treatments at the time of presentation were mixed in wells, with observation of precipitate formation. Precipitates were observed with phasecontrast microscopy, and subsequently characterised via crystallography techniques and electrospray ionisation mass spectrometry.
\end{abstract}

Results Combinations of dexamethasone $\mathbf{0 . 1 \%}$ minims and chlorhexidine gluconate $0.2 \%$ formed an amorphous material characterised by electrospray ionisation mass spectrometry as an insoluble chlorhexidine salt. Combinations of chloramphenicol drops and timolol $0.5 \%$ formed a crystal identified via X-ray crystallography as santite $\left(\mathrm{K}\left(\mathrm{B}_{5} \mathrm{O}_{6}(\mathrm{OH})_{4}\right) \cdot\left(\mathrm{H}_{2} \mathrm{O}\right)_{2}\right)$. This is a borate mineral identified in nature, arising from thermal springs, but never reported in biological tissues. Clinical improvement was observed following the cessation of the implicated precipitating combinations. Conclusion Our observations suggest iatrogenic precipitate formation, with a potential deleterious effect upon healing. The substrates for these precipitates include several frequently prescribed topical ophthalmic treatments. These findings shed new light on the aetiopathogenesis of nonhealing corneal ulceration, and have broad implications on topical prescribing for this challenging condition.
I Livingstone', F Stefanowicz², S Moggach³,

J Connolly', S Ramamurthi', S Mantry ${ }^{1}$ and

K Ramaesh
Eye (2013) 27, 755-762; doi:10.1038/eye.2013.39; published online 5 April 2013

Keywords: acanthamoeba; corneal ulcer; santite; chlorhexidine

\section{Introduction}

Non-healing corneal ulcers are challenging clinical entities. The aetiology of such ulcers are multifactorial, including persistence of infection, treatment toxicity, steroid use, neurotrophic keratopathy, dry eye, exposure keratopathy, and chronic conjunctival inflammation in such conditions as ocular cicatricial pemphigoid and atopic keratoconjunctivitis. In this case series, we present a novel observation where interaction of topical treatments may have contributed to persistence of corneal ulceration in the context of previous culture-proven acanthamoeba keratitis. Crystallographic techniques allowed characterisation of the precipitate in one case, demonstrating a unique crystalline compound, not previously identified in the context of corneal disease, arising from the interaction of two commonly prescribed topical treatments.

\section{Materials and methods}

We report two cases presenting to our corneal service. Both cases involved eyes with a background of acanthamoeba keratitis, and were characterised by persistent epithelial defects with the appearance of a crystalline deposition throughout the stroma.

\section{Confocal microscopy}

Following topical anaesthesia (Minims proxymetacaine $0.5 \%$, Bausch \& Lomb, Kingston upon Thames, UK), scanning-slit invivo confocal microscopy was carried out
${ }^{1}$ Tennent Institute of Ophthalmology, Gartnavel General Hospital, Glasgow, UK

${ }^{2}$ Scottish Trace Element and Micronutrient Reference Laboratory, Department of Clinical Biochemistry, Glasgow Royal Infirmary, Glasgow, UK

${ }^{3}$ School of Chemistry, The University of Edinburgh, Edinburgh, UK

Correspondence: I Livingstone, Tennent Institute of Ophthalmology, Gartnavel General Hospital, 1053 Great Western Road, Glasgow G12 OYN, UK. Tel: + 44 (0)7595776726; Fax: + 44 (0)141 2112054 ; E-mail: iainatlivingstone@ gmail.com

Previously presented: Oxford Congress (poster presentation) July 2011. Scottish Ophthalmology Club Centenary Meeting (poster presentation) September 2011. Financial Disclosure: This work won 1st Place in the Pfizer/SOC Travel Prize. A travel grant of $£ 1500$ was awarded to lain Livingstone.

Received: 29 March 2012 Accepted in revised form: 6 February 2013 Published online: 5 April 2013 
(Confoscan 4, Nidek Technologies, Albignasego, Italy). The contact $\times 40$ lens was used with a coupling gel (Viscotears, Novartis, Frimley, UK) and the full-thickness option on the instrument was selected. The instrument focuses on the bright reflex of the endothelium and scans the cornea in a posterior-to-anterior direction. Images were obtained from the central and paracentral cornea.

\section{Microbiology}

To investigate the aetiology of the non-healing ulceration, viral swabs as well as repeat corneal scrapes were performed for microbiological analysis for both patients.

\section{Precipitation}

To investigate possible precipitating combinations, all concurrent treatments at the time of presentation were mixed in wells, with observation of precipitate formation. These were collected, and subjected to phasecontrast microscopy, and crystallographic techniques.

\section{Single X-ray diffraction}

Single-crystal X-ray diffraction data were collected at $100 \mathrm{~K}$ using an Oxford Cryosystems (Oxford, UK) low temperature device attached to an Oxford Diffraction SuperNova dual wavelength diffractometer equipped with an Atlas (Oxford Diffraction, Abingdon, UK) charged coupled device (CCD) detector and operating in $\mathrm{CuK}_{\alpha}$ radiation mode $(\lambda=1.54184 \AA)$. The structure was solved using direct methods (Sir92). Refinement was carried out against $|F|^{2}$ using all data.

\section{Electrospray ionisation mass spectrometry (ESI-MS)}

Amorphous, non-crystalline precipitate was characterised using electrospray ionisation mass spectrometry (ESI-MS) in positive ion mode by infusion analysis (direct ESI-MS). The precipitate was isolated from solution by centrifugation $\left(500 \mathrm{~g}, 4^{\circ} \mathrm{C}, 10 \mathrm{~min}\right)$. The supernatant was decanted and the pellet was redissolved in $18 \mathrm{M} \Omega$ deionised water to give a $0.1 \mathrm{mg} / \mathrm{ml}$ solution. The topical treatment provided by pharmacy comprised
$0.2 \mathrm{mg} / \mathrm{ml}$ chlorhexidine digluconate in deionised water and was used directly as a standard for comparison. The redissolved precipitate and standard compound were directly introduced into an ACQUITY TQ Detector mass spectrometer via an ESI probe and onboard fluidics system (Waters, Milford, MA, USA). Solutions were infused at a rate of $5 \mu \mathrm{l} / \mathrm{min}$. The data were acquired using Waters MassLynx software (version 4.1). The capillary and cone voltages were set at $1 \mathrm{kV}$ and $+40 \mathrm{~V}$, respectively. The source block and desolvation temperatures were set at 150 and $500{ }^{\circ} \mathrm{C}$. The nebuliser and cone gas flow rates were set at 1000 and 5001/h, respectively. MS data were collected as total ion current.

\section{Results}

\section{Case 1}

A 68-year-old female patient presented with recalcitrant epithelial and stromal defects affecting the left eye. Her complex past ophthalmic history included biopsyproven contact lens-associated acanthamoeba keratitis 10 years previously, complicated by scleritis, progressing to corneal perforation, ultimately necessitating penetrating keratoplasty (PK). She developed anterior scleritis in the postoperative period, with subsequent graft failure necessitating regrafting. Augmented trabeculectomy with mitomycin $\mathrm{C}$ was performed to control persistently elevated intraocular pressure 3 years later. Corrected visual acuity at this stage was measured at $6 / 5$. More recently, the second graft showed signs of rejection with bullous keratopathy and raised intraocular pressure. At the time of presentation to our service, topical treatments comprised timolol maleate $0.5 \%$ BD and chloramphenicol $0.5 \%$ QDS. Visual acuity in the affected left eye had declined to 'hand movements'. There was diffuse conjunctival injection, perilimbal neovascularisation, pancorneal oedema, and an inferior non-healing epithelial defect. High-magnification slitlamp biomicroscopic examination $(\times 40)$ showed tiny reflective bodies in anterior stroma, with appearances suggestive of crystal formation (Figure 1a).

Confocal microscopic examination evidenced multiple crystalline-like deposits within the corneal stroma

Figure 1 (a) Case 1 slit-lamp photograph showing predominantly superior corneal neovascularisation, diffuse corneal oedema and localised infiltrate. Reflective bodies were evident within the anterior stroma, concentrated around the edges of the epithelial defect. (b) Case 1 slit-lamp photographs with fluorescein staining, evidencing well-demarcated epithelial defect. (c) Case 1, confocal microscopy evidencing highly refractive crystal-like entities, concentrated within the anterior stroma. (d and e) Case 1 appearances 2 weeks after cessation of gutte chloramphenicol/gutte timolol combination. Despite continued corneal oedema and persistent infiltrate, there is near complete closure of the epithelial defect. (f) Case 2 slit-lamp photograph showing diffuse infiltrate around a persistent epithelial defect. Tiny reflective bodies were evident in the anterior stroma. (g) Case 2 slit-lamp photograph with fluorescein staining, evidencing large epithelial defect. (h) Case 2 confocal microscopy, evidencing highly refractive entities, concentrated within the anterior stroma within the constraints of the epithelial defect. 


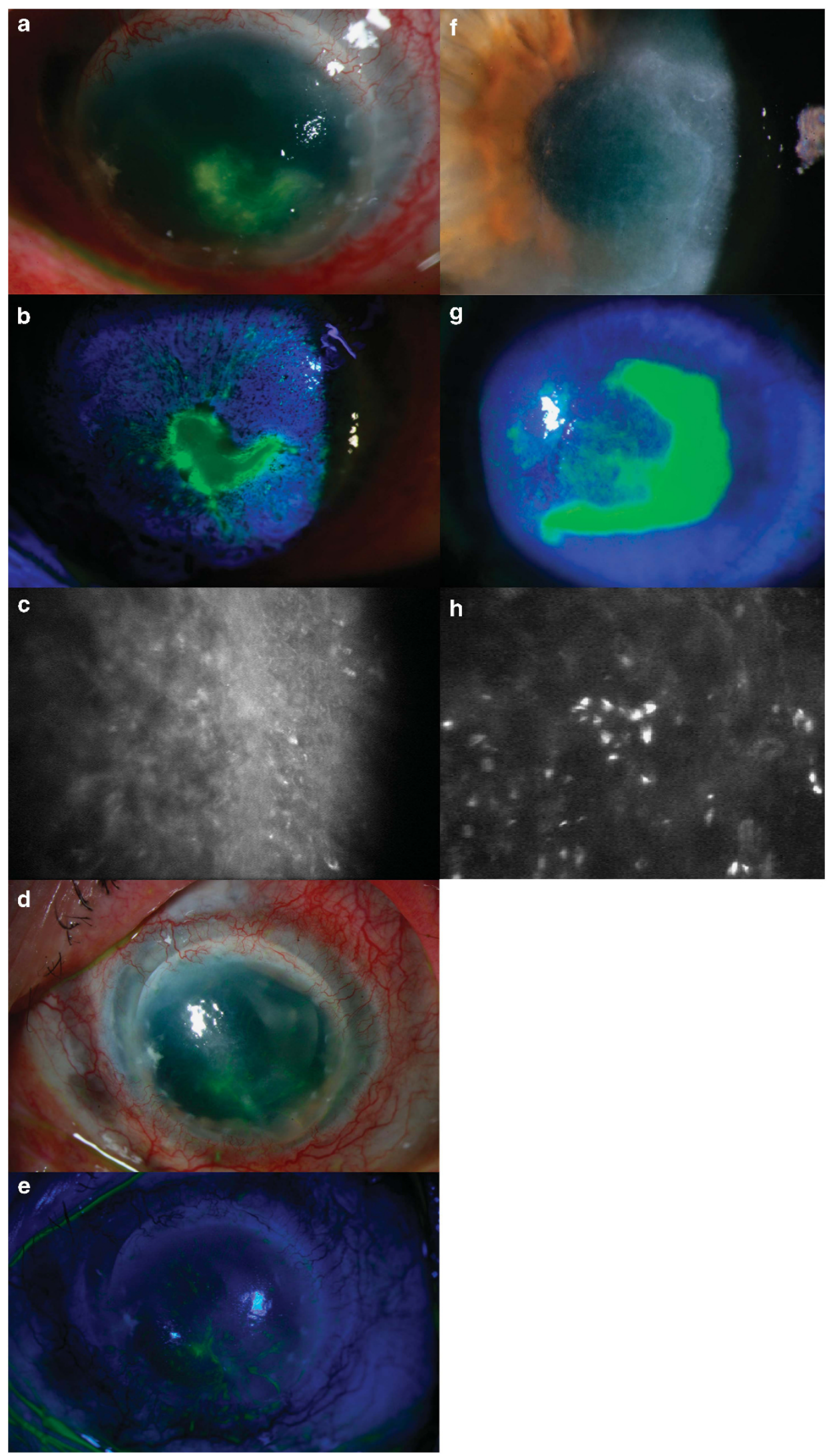


a

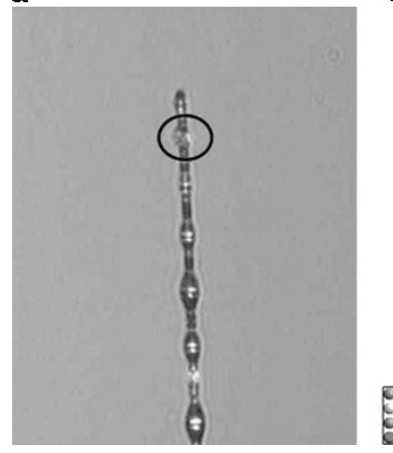

b

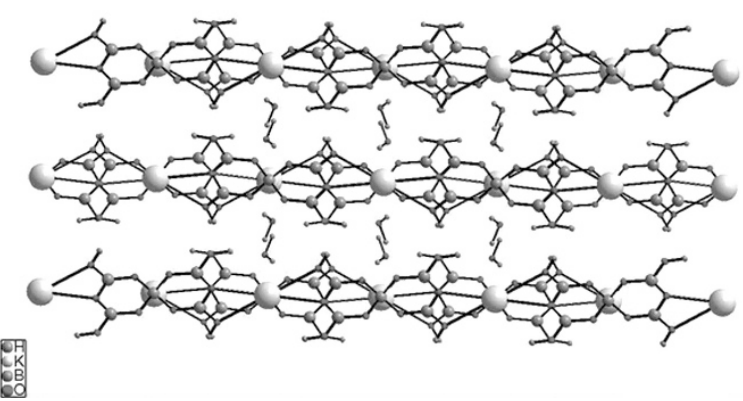

Figure 2 (a) Photograph of a crystal of santite from the timolol/chloramphenicol eye drop mixture mounted on a glass fibre for single-crystal X-ray diffraction. The photograph shows the formed crystal (circled), $8 \mu \mathrm{m}$ in diameter. (b) The crystal structure as viewed down the [100] direction.

(Figure 1c). Neither acanthamoeba cysts, nor perineuritis were evidenced. Precipitation of agents within topical ophthalmic treatments was suspected, and the treatment regime of chloramphenicol and timolol were discontinued. Doxycycline $100 \mathrm{mg}$ OD and lacrilube ointment nocte were commenced. Figure $1 \mathrm{~d}$ shows appearances after 2 weeks of discontinuing chloramphenicol and timolol. Repeat corneal scrape gram stain, bacterial/fungal cultures and viral swabs yielded negative results.

Concurrent ophthalmic treatments were mixed in vitro, and were examined with phase-contrast microscopy. We observed crystalline precipitate on combination of timolol maleate and chloramphenicol eye drops. The precipitate was collected and subject to phase-contrast microscopy, and then X-ray diffraction crystallography techniques as described.

Case 1: crystallography results Using crystallography techniques, the precipitate was characterised as santite, a mineral formally called potassium closotetrahydroxohexaoxopentaborate dehydrate $\left(\mathrm{K}\left(\mathrm{B}_{5} \mathrm{O}_{6}(\mathrm{OH})_{4}\right) \cdot\left(\mathrm{H}_{2} \mathrm{O}\right)_{2}\right)$. Figure 2a shows the formed santite crystal, mounted on a glass fibre, with the structure illustrated in Figure $2 b$. Santite is found as a naturally occurring borate mineral named after Italian chemist Giorgio Santi in 1970. It occurs rarely in nature, and forms in fumaroles, thought to arise from reaction between postassium-bearing hot solutions and deposits of lardellite, forming around thermal springs, localised from thermal springs in Larderello, Italy and Eagle Borax Spring, Death Valley, CA, USA. ${ }^{1-3}$

\section{Case 2}

A 21-year-old female soft contact lens-wearer presented with culture-proven unilateral acanthamoeba keratitis. She was initially treated with polyhexamethylene biguanide (PHMB) and chlorhexidine, and preservativefree $0.1 \%$ dexamethasone minims were added 4 weeks later. This treatment was effective initially, but after 6 weeks, the clinical picture deteriorated (Figures $1 \mathrm{f}$ and g). Further corneal scrapes were negative for fungi, virus, and bacteria. Confocal microscopy also suggested crystal-like material within the corneal stroma (Figure 1h), with no evidence of perineuritis or cysts. Once again, a precipitation reaction between topical treatments was suspected, and all current topical treatments were stopped. Pred forte (prednisolone acetate $1 \%$ ophthalmic solution, Allergan, Marlow, UK) was commenced.

On mixing all possible permutations of the various concurrent topical treatments in vitro, crystal-like precipitates were only detected in combinations of chlorhexidine (chlorhexidine gluconate $0.2 \%$ ) and dexamethasone (dexamethasone $0.1 \%$, preservative-free). PHMB did not form precipitate when mixed with chlorhexidine or dexamethasone. No agent in isolation formed precipitate. The amorphous material arising from chlorhexidine and dexamethasone was not crystalline, and underwent mass spectrometry for characterisation. While improvement was observed on discontinuation of the suspected offending combination, amniotic membrane graft was ultimately performed to encourage healing.

Case 2: mass spectrometry results Mass spectra of the chlorhexidine standard is shown in Figure 1f. The peak observed at $m / z 505$ represents the protonated molecular ion $\left(\mathrm{M}+\mathrm{H}^{+}\right)$. A further, less-abundant peak is observed at $m / z 507$ due to the presence of two chlorines in the structure of chlorhexidine. The ratio of the peak heights of $\mathrm{m} / \mathrm{z} 505$ and 507 is characteristic of the ratio of chlorine isotopes. A further molecular ion-related peak is observed at $\mathrm{m} / \mathrm{z} 253$ and represents the doubly charged molecular ion $(\mathrm{M}+2 \mathrm{H})^{2+}$. Peaks present at $m / z 353,336$, 


\section{a}

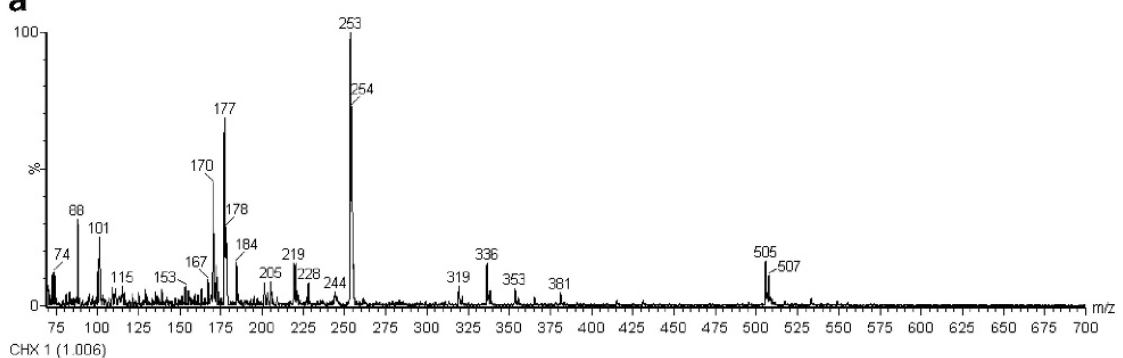

b

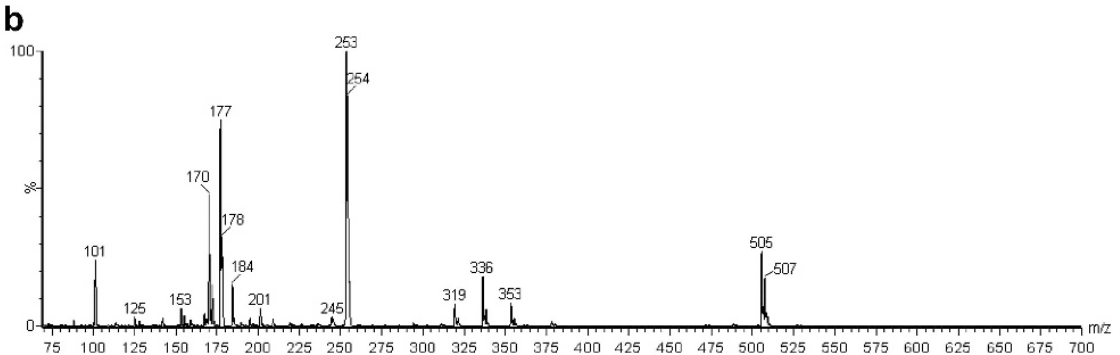

Figure 3 Electrospray ionisation mass spectrometry results for chlorhexidine/dexamethasone $0.1 \%$ minims (a), and chlorhexidine alone (b). The two highest peaks in both spectra reflect the molecular ions for chlorhexidine (CHX). The molecular mass of CHX is 504 . Using ESI as an ion source, the compound encompasses a proton, increasing the molecular mass by +1 . The two peaks at 505 and 507 reflects the two $\mathrm{Cl}$ atoms present in the structure which have isotopes at 35 (75\% total abundance) and 37 (25\% total abundance). Peaks with lower masses represent fragments of the molecule as it breaks apart in the ion source, and are compound characteristic. It can be seen that the top panel (precipitate) exactly matches that of chlorhexidine. The few extra small peaks reflect contamination.

177 and 170 represent fragment ions, the structure of which have been described elsewhere. ${ }^{4}$ The molecular ion, isotopic ratio, doubly charged molecular-related ion and fragment ions are all discriminating features useful in characterising chlorhexidine. The mass spectra of the redissolved precipitate (Figure $3 \mathrm{~b}$ ) was found to mirror the features present in that of the chlorhexidine standard confirming the identity of the precipitant to be chlorhexidine.

\section{Discussion}

Our observations suggest that generation of crystal-like deposits was iatrogenic, precipitating from the given combinations. These precipitates appear to have persisted in the de-epithelialised stroma. In both cases, discontinuing the proposed offending combinations led to clinical improvement. Continued acanthamoeba infection and bacterial superinfection were excluded with repeated negative corneal scrapes. Confocal microscopy failed to show acanthamoeba trophozoytes or cysts, but disclosed the presence of crystal-like opacities at various levels within the stroma. These opacities were visible under high-magnification slit-lamp biomicroscopy. Our clinical suspicion was supported by a simple in-vitro study, where mixing of chloramphenicol with timolol, and dexamethasone with chlorhexidine, formed precipitates similar in appearance to those demonstrated within the non-healing area of ulceration. Characterisation of the resulting precipitates was undertaken. The precipitate formed on mixing timolol with chloramphenicol was found to be crystalline and was characterised by X-ray crystallography, identified as the mineral santite. Although the precipitate has been successfully identified, the presence and source of the $\mathrm{B}_{5} \mathrm{O}_{6}(\mathrm{OH})_{4}{ }^{-}$anion, which rarely occurs in nature, is still unknown and remains a subject for further investigation.

The chlorhexidine/dexamethasone combination appeared to form an amorphous precipitate, and it was not possible to further characterise its structure via crystallographic techniques. Molecular mass spectrometry was therefore used to identify the precipitate. ESI was chosen as an ion source, as it is a suitable technique for the detection of compounds that lack volatility and thermal stability. Comparison of mass spectra revealed the identity of the amorphous precipitate to be chlorhexidine. Chlorhexidine is a cationic molecule that is able to form sparingly soluble salts with anions such as phosphate, sulphate and carboxylates. ${ }^{5-8}$ Consequently, pharmacies dispense chlorhexidine in the highly soluble form of chlorhexidine digluconate dissolved, exclusively, in pure water. Dexamethasone minims contain many phosphates in the form of buffers. It is our theory that on combining the chlorhexidine preparation with the dexamethasone minims, an interaction occurs between the chlorhexidine 
and phosphates, thereby reducing the solubility of chlorhexidine resulting in precipitation.

Such findings have broad implications for the role of chlorhexidine and polypharmacy for acanthamoeba keratitis, paving the way for further studies to investigate the efficacy of this agent when used in combination with other drops, and its propensity for precipitation and potential toxicity.

The mechanism of toxicity has yet to be elucidated, though there are notable examples where crystal formation causes damage to biological tissues, such as pneumoconioses, crystal arthropathies, osteoarthritis, and calcific tendonitis. ${ }^{9}$ These disease processes vary in pathology, but share features of degenerative proteases, cytokines, chemokines, and prostanoids produced by cells stimulated by crystals. ${ }^{9}$ We suspect that precipitation of santite and the chlorhexidine salt contributed to surface toxicity, potentiating keratitis and epithelial devitalisation. It should be noted however, that the toxicity of the drops in isolation cannot be excluded as contributing factors, given that prolonged use of topical, preserved drugs have long been shown to cause ocular surface inflammation ${ }^{10,11}$ and dry eye syndrome. ${ }^{12}$ Furthermore, the recovery of non-healing defects on cessation of previous topical therapy is a well-known phenomenon. In the case of the santite crystal and chlorhexidine salt, the potential for pathologic effects requires further study.

The literature contains various examples of iatrogenic crystal formation secondary to drop precipitation, primarily with the fluroquinolones. ${ }^{13-18}$ SchlotzerSchrehardt et al ${ }^{19}$ reported corneal stromal calcium deposition in all levels of the stroma, secondary to topical dexamethasone phosphate therapy in the context of chronic keratoconjunctivitis in a patient with StevensJohnson syndrome. In the latter study, histopathological analysis of the corneal button evidenced calcium deposits closely associated with intracellular and pericellular accumulations of glycosaminoglycans, suggesting a possible role of dexamethasone phosphate treatment altering the glycosaminoglycan metabolism of stromal keratocytes in the calcification process. ${ }^{19}$

Huige et $a^{20}$ observed superficial stromal precipitation of calcium phosphate in a series of eight patients. Cited predisposing factors in all cases include epithelial defect, with combined use of topical dexamethasone

phosphate/prednisolone phosphate (two out of the eight patients were given preservative-free preparations), together with topical beta-blockers. In common with our series, discontinuation of the combination led to clinical improvement. These authors theorised that calcium phosphate gained access to the stroma via defects in the epithelium. In a similar series, Lake et $a l^{21}$ describe six patients with deep corneal calcification in the context of persistent epithelial defect and high-phosphate content preservative-free topical treatment (timolol, dexamethasone, and prednisolone).

Taravella et $a l^{22}$ reported five cases of calcium deposition in the cornea associated with the use of topical steroid phosphate preparations. Common characteristics include multiple topical treatments and the presence of epithelial defect.

Throughout the literature, corneal calcification most typically occurs as calcium phosphate deposition. ${ }^{19,23}$ Bernauer et $a l^{24}$ reported potential adverse effects of intensified sodium hyaluronate artificial tears in patients with various ocular surface conditions. The authors describe five cases of deep corneal calcium crystal deposition, ultimately requiring keratoplasty. The crystalline deposits were found to consist of hydroxyapatite, $\mathrm{Ca}_{5}\left(\mathrm{PO}_{4}\right)_{3} \mathrm{OH}$. Light microscopy of the corneal buttons demonstrated partial loss of epithelium with mineralisation of Bowman's membrane and the entire stroma. The calcification reached and involved Descemet's membrane in four out of the five samples. A mild inflammatory infiltrate was present in three samples, though no microorganisms were detected. The sodium hyaluronate preparation implicated as the cause was found to contain a 50-fold higher phosphate content than normal serum, and significantly higher phosphate content than alternative preparations. The topical application of drops containing high phosphate levels has been linked to the development of band keratopathy, ${ }^{19}$ albeit without inciting an apparent inflammatory response.

There is growing clinical evidence demonstrating that the electrolyte content of intensive drops contributes to deposition of insoluble salts in the corneal stroma. The risk apparently increased when such drops are applied in the context of ocular surface disease. ${ }^{25}$

We postulate that iatrogenic formation of crystals or crystal-like precipitates cause direct damage to cellular structures, causing corneal toxicity, potentiating epithelial defects. The cases reported in our study share a background of acanthamoeba keratitis, and the localisation of the crystals to the area of de-epithelialisation may reflect a required substrate within exposed stroma. Definitive characterisation of the crystal-like opacities seen in both patients would require harvesting of a sample directly from the corneal stroma, though, even if such invasive methods were employed, it is unlikely that the required volume of material would be sufficient to undergo the identification techniques described. Nevertheless, in vivo, the biological ecosystem encompasses multiple agents absent in vitro, with factors such as $\mathrm{pH}$, immune cells and their derivatives, damaged cells and components of the tear-film likely having a role.

In the case of the santite crystal, this is the first report of a potentially toxic interaction between two 
well-established and common topical treatments. The concurrent use of chloramphenicol and timolol in the context of a compromised ocular surface is likely to represent a relatively common scenario, making the present findings surprising. The increased availability of confocal microscopy proved helpful in viewing the entities in more detail than was possible with slit-lamp biomicroscopy, prompting further study. Furthermore, it is widely accepted that ophthalmic polypharmacy can contribute to ocular surface toxicity, and the practice of drop cessation in the case of persistent epithelial defect is common. Had the decision to stop all drops been made earlier, the preciptates may not have increased to the degree that they were detectable on slit-lamp biomicroscopy. Other circumstances specific to this series include the prior history of acanthamoeba, and inherent long history of compromised ocular surface. In addition, in case 1 , the topical treatments continued over a prolonged period of $>6$ months, theoretically contributing to a significantly greater density of santite crystals. We suspect the incidence of precipitant toxicity is more common than is currently appreciated, and it is our hope that by highlighting such observations and characterising the precipitants, these index cases will raise awareness of the propensity for such interactions.

\section{Conclusion}

In this study, we have demonstrated the novel formation of two different types of precipitates from common ophthalmic treatments. First is the crystal santite, arising from the interaction of timolol and chloramphenicol. The second is the amorphous glass-like precipitation of chlorhexidine, formed via interaction of anions present in the dexamethasone minims.

The potential for these common ophthalmic topical treatments to interact and form toxic products has broad implications on topical polypharmacy in ophthalmic practice. Our findings highlight the need for future toxicological studies in this area.

\section{Summary}

What was known before

- Several classes of topical treatments have been known to form precipitate within the cornea with deleterious effect.

\section{What this study adds}

- In vitro, interaction of common ophthalmic preparations form a crystalline compound that that has not been identified in biological tissues. Such precipitants may contribute to persistent epithelial defects when deposited in vivo. This study raises awareness of the potential for iatrogenic damage in ophthalmic polypharmacy.

\section{Conflict of interest}

The authors declare no conflict of interest.

\section{References}

1 Merlino S, Sartori F. Santite, a new mineral phase from Larderello, Tuscany. Can Mineral 1970; 27: 159-165.

2 Cook WR, Jaffe H. The crystallographic, elastic, and piezoelectric properties of ammonium pentaborate and potassium pentaborate. Acta Cryst 1957; 10: 705-707.

3 Clark JR, Christ CL. Studies of borate minerals (VII): X-ray studies of ammonioborite, larderellite, and the potassium and ammonium pentaborate tetrahydrates. Amer Mineral 1959; 44: 1150-1158.

4 Usui K, Takanori H, Yamaguchi H, Tachiirib N, Goto J. Determination of chlorhexidine (CHD) and nonyphenolethoxylates (NPEOn) using LC-ESI-MS method and application to hemolysed blood. J Chrom B 2006; 831: 105-109.

5 Barkvoll P, Rølla G, Bellagamba S. Interaction between chlorhexidine digluconate and sodium monofluorophosphate in vitro. Scand J Dent Res 1988; 96: 30-33.

6 Rølla G, Loe H, Schiott CR. The affinity of chlorhexidine for hydroxyapatite and salivary mucins. J Periodont Res 1970; 5: 79-83.

7 Rølla G, Melsen B. On the mechanism of the plaque inhibition by chlorhexidine. J Den Res 1975; 54: 1357-1362.

8 Bonesvoll P. Influence of ionic strength, calcium, sodium, dodecyl sulphate and urea on the retention of chlorhexidine in the human mouth after mouth rinses. Arch Oral Biol 1977; 22: 273-279.

9 Molloy ES, McCarthy GM. How crystals damage tissue. Curr Rheumatol Rep 2004; 6(3): 228-234.

10 Becquet F, Goldschild M, Moldovan MS, Ettaiche M, Gastaud P, Baudouin C. Histopathological effects of topical ophthalmic preservatives on rat corneoconjunctival surface. Curr Eye Res 1998; 17(4): 419-425.

11 Mietz H, Niesen U, Krieglstein GK. The effects of preservatives and antiglaucomatous medication on histopathology of the conjunctiva. Graefes Arch Clin Exp Ophthalmol 1994; 232(9): 561-565.

12 Gobbels M, Spitznas M. Corneal epithelial permeability of dry eyes before and after treatment with artificial tears. Ophthalmology 1992; 99(6): 873-878.

13 Castillo A, Benitez del Castillo JM, Toledano N, Diaz-Valle D, Sayagues O, Garcia-Sanchez J. Deposits of topical norfloxacin in the treatment of bacterial keratitis. Cornea 1997; 16(4): 420-423.

14 Konishi M, Yamada M, Mashima Y. Corneal ulcer associated with deposits of norfloxacin. Am J Ophthalmol 1998; 125(2): 258-260.

15 Eiferman RA, Snyder JP, Nordquist RE. Ciprofloxacin microprecipitates and macroprecipitates in the human corneal epithelium. J. Cataract Refract Surg 2001; 27(10): 1701-1702.

16 Claerhout I, Kestelyn P, Meire F, Remon JP, Decaestecker T, Van Bocxlaer J. Corneal deposits after topical use of ofloxacin in two children with vernal keratoconjuctivitis. Br J Ophthalmol 2003; 87(5): 646.

17 Sinnaeve BA, Decaestecker TN, Claerhout IJ, Kestelyn P, Remon JP, Van Bocxlaer JF. Confirmation of ofloxacin 
precipitation in corneal deposits by microbore liquid chromatography-quadrupole time-of-flight tandem mass spectrometry. J Chromatogr B 2003; 785(1): 193-196.

18 Awwad ST, Haddad W, Wang MX, Parmar D, Conger D, Cavanagh HD. Corneal intrastromal gatifloxacin crystal deposits after penetrating keratoplasty. Eye Contact Lens Sci Clin Pract 2004; 30(3): 169-172.

19 Schlotzer-Schrehardt U, Zagorski Z, Holbach LM, Hofmann-Rummelt C, Naumann GO. Corneal stromal calcification after topical steroid-phosphate therapy. Arch Ophthalmol 1999; 117(10): 1414-1418.

20 Huige WMM, Beekhuis WH, Rijneveld WJ, Schrage N, Remeijer L. Unusual deposits in the superficial corneal stroma following combined use of topical corticosteroid and beta-blocking medication. Doc Ophthalmol 1991; 78(3-4): 169-175.
21 Lake D, Tarn A, Ayliffe W. Deep corneal calcification associated with preservative-free eyedrops and persistent epithelial defects. Cornea 2008; 27(3): 292-296.

22 Taravella MJ, Stulting RD, Mader TH, Weisenthal RW, Forstot SL, Underwood LD. Calcific band keratopathy associated with the use of topical steroid-phosphate preparations. Arch Ophthalmol 1994; 112(5): 1475.

23 Daly M, Tuft SJ, Munro PM. Acute corneal calcification following chemical injury. Cornea 2005; 24(6): 761-765.

24 Bernauer W, Thiel MA, Kurrer M, Heiligenhaus A, Rentsch $\mathrm{KM}$, Schmitt A et al. Corneal calcification following intensified treatment with sodium hyaluronate artificial tears. Br J Ophthalmol 2006; 90(3): 285-288.

25 Mukherjee S, O'Reilly DS, Cox A, Ramaesh K. Phosphate and electrolyte content of topical steroid drop and potential clinical implications. Br J Ophthalmol 2008; 92(4): 574-576. 